${ }^{2}$ Coulthard MG, Hey EN, Ruddock V. Creatinine and urea clearances compared to inulin clearance in preterm and mature babies. Early Hum Dev 1985;11:11-9.

M G Coulthard, E N Hey, and V Ruddock Central Sector,

Royal Victoria Infirmary, Queen Victoria Road, Newcastle upon Tyne NE1 4LP

\section{Patient triggered ventilation in premature neonates}

Sir,

Greenough and Greenhall ${ }^{1}$ recently reported their experience of the trigger ventilator. ${ }^{2}$ They modified our original circuit by switching the inspiration detector from its site on the abdomen to the oesophagus. We had considered this approach but felt that it had a limited role because it could not be used on a routine basis by untrained staff. In their paper they allege that 'gross body movements' made the abdominal sensor prone to false triggering. This has never been a problem in my experience over the last three years. While it is obviously theoretically possible to interfere with the signal from the capsule, in practice the design of the circuit largely eliminates this problem. This is best illustrated by the example of an infant on triggered ventilation with an inspiratory time set at its recommended 0.5 seconds. When a breath occurs there is a delay of up to $100 \mathrm{msec}$ before the trigger fires. The ventilator is refractory to further signals from the time of firing, through the inspiratory time setting ( 0.5 seconds) and for 0.25 seconds after this interval (to allow enough time for expiration). Most infants breathe at 60 breaths (approximately) per minute with this inspiratory time (unpublished work). Thus on average the ventilator is refractory for 0.85 seconds out of the 1 second between breaths and only 0.15 seconds remain as a susceptible period before the next breath is due. The only consequence of such a false signal is to make the next breath occur fractionally early.

I submit therefore that the criticism of our original approach is invalid and that triggered ventilation using an abdominal sensor is an effective means of assisting the newborn's respiratory efforts. ${ }^{3}$

\section{References}

${ }^{1}$ Greenough A, Greenall F. Patient triggered ventilation in premature neonates. Arch Dis Child 1987;63:77-8.

2 Mehta A, Wright BM, Callan K, Stacey TE. Patient triggered ventilation in the newborn. Lancet 1986;ii:17-9.

${ }^{3}$ Clifford RD, Whincup G, Thomas R. Patient-triggered ventilation prevents pneumothorax in premature babies. Lancet 1988;i:529-30.

A Mehta

Department of Paediatrics, School of Medicine,

University College London,

The Rayne Institute, University Street, London WC1E 6JJ

\section{Infective endocarditis in neonates}

Sir,

We read with interest the recent review of five patients with neonatal infective endocarditis by $\mathrm{O}^{\prime}$ Callaghan and McDougall. ${ }^{1}$ We wish to report a further case where echocardiography had an essential role in the early diagnosis of pericarditis and endocarditis in a newborn infant where clinical signs specific to endocarditis were absent.

A boy weighing $3200 \mathrm{~g}$ was born vaginally at term to a primagravida who had loose bloody stools due to Shigella sonnei infection during the last trimester of pregnancy. The infant developed shigella enteritis with intermittent gaseous abdominal distension. Ultrasound examination showed an abdominal abscess but during this procedure the pericardial effusion was incidentally detected. Although the infant was ill and looked septic, arterial pulses and blood pressure were normal. The heart sounds were normal with no evidence of a murmur or pericardial rub. The chest radiograph showed cardiomegaly but the electrocardiogram was normal. A septic spot was noted on the sternum. A peripheral blood count showed chromocytopenia but no petechial lesions were present. There was no haematuria.

Cross sectional echocardiography confirmed the presence of a large anterior pericardial effusion as well as a vegetation on the anterior leaflet of the mitral valve. Thirty $\mathrm{ml}$ of pus was obtained by needle aspiration of the pericardial space. Subsequent surgical exploration was performed and the pericardial space was left on continuous drainage for one week. An identical strain of Staphylococcus aureus was isolated from the pericardial fluid, blood cultures, and the septic lesion on the sternum. Gentamicin and cloxacillin to which the organism was sensitive were given intravenously for six weeks. After one week of treatment the pericardial effusion had resolved but the vegetation persisted. Umbilical arterial or venous lines were not used at any time during his management. He was discharged from hospital at the age of 3 months. At that time his cardiac examination was normal; in particular there was no evidence of mitral valve insufficiency. Echocardiography confirmed persistence of the mitral valve vegetation which had become more echodense and possibly calcified. After discharge from hospital the infant was lost to follow up.

Clinical detection of bacterial endocarditis in the newborn who is already ill from sepsis is difficult. Embolic phenomena, congestive cardiac failure, and changing heart murmurs are important clues but are infrequently found. ${ }^{2}$ Cross sectional echocardiography permits earlier diagnosis of endocarditis provided vegetations are at least $2 \mathrm{~mm}$ in diameter. ${ }^{2}$ We surmise that survival of our patient was due to early diagnosis and prompt treatment with appropriate antibiotics in high doses, and the maintenance of normal tissue perfusion and blood pressure in the presence of overwhelming septicaemia. We concur with O'Callaghan and McDougall in the usefulness of echocardiography in the diagnosis of neonatal endocarditis. We have shown that with early diagnosis recovery is possible. 